\title{
MUJERES EN ESCENA. PATRICIA DAVIS Y LA BARCA OTRO TEATRO
}

\author{
WOMEN ON STAGE. PATRICIA DAVIS AND LA BARCA OTRO TEATRO
}

Laura Hernández Lorenzo

Universidad de Sevilla

\section{ReSUMEN:}

Este artículo pretende reivindicar la profesionalidad de la mujer en las artes escénicas. Patricia Davis es psicóloga social y directora teatral de La Barca Otro Teatro y Espontagas. La actriz es experta en improvisación y pretende concienciar a la sociedad sobre la importancia de la educación en la igualdad de género.

\section{Palabras claves:}

Artes escénicas, Improvisación, Patricia Davis, Género.

\section{Abstract:}

This article seeks to reclaim the professionalism of women in the performing arts. Patricia Davis is a social psychologist and the theatre director of La Barca Otro Teatro and Espontagas. She is an expert in improvisation and works to raise social awareness about the importance of education in gender equality.

\section{KEY WORD:}

Performing arts, Improvisation, Patricia Davis, Gender. 


\section{UNA MUJER POLIFACÉTICA}

Patricia Davis nace en Argentina y crece en un barrio humilde en el extrarradio de Buenos Aires. Tras finalizar sus estudios de secundaria (a la edad de 19 años), comenzó a trabajar con grupos de jóvenes en promoción de la salud y participó en numerosos encuentros; gracias a ello, le concedieron una beca para trabajar en la Secretaría de Prevención y Asistencia de las Adicciones de la Provincia de Buenos Aires. Davis compaginó esta etapa profesional con su formación teatral y ejerció esta actividad desde 1995 hasta 2000.

La institución estaba compuesta por profesionales, psicólogos, sociólogos y trabajadores sociales. Esta experiencia llegó a ser muy enriquecedora para ella. El organismo creó un programa para jóvenes, y finalmente, la dirección provincial nombró a Davis coordinadora de formación. Dos años más tarde, tras un cambio en la gestión, los nuevos directivos le ofrecieron trabajar en prevención comunitaria. En esta etapa (1998-2002), ella había empezado a estudiar Psicología Social, puesto que se sentía apasionada por el trabajo en grupos. En el año 2001 conoce por primera vez la capital hispalense pero decide volver a Argentina. Un año después, en mayo de 2002, la Dirección Nacional de Juventud la nombró responsable de la Coordinación Nacional de Planificación y Capacitación para Jóvenes. Gracia a este cargo, tuvo la oportunidad de trabajar con miles de personas en todo el país, diseñar estrategias de intervención, programas de capacitación y formar equipos técnicos. Ese año visitó Sevilla por segunda vez; fue entonces cuando Davis colaboró en temas de logística junto a la que había sido su directora de teatro en Argentina que había fundando la compañía Dos Lunas y organizado un Encuentro Internacional de Mujeres en las Artes Escénicas. A partir de ese momento, decidió permanecer en Sevilla con el propósito de establecer disciplinas como el teatro espontáneo y la improvisación. ${ }^{1}$ En esta nueva etapa, la psicóloga social comenzó trabajando con la compañía Dos Lunas y posteriormente fundó La Barca Otro Teatro.

\section{LA INCORPORACIÓN DE DAVIS A LA EMPRESA TEATRAL}

1 Davis se ha formado internacionalmente con profesionales como Jacobo Moreno (teatro espontáneo), Enrique Pichón Riviere, (psicología social) Eduardo Galeano, por su prosa poética cargada de compromiso social, Idea Vilariño, por su capacidad de síntesis poética y Rolando Toro, creador de la Biodanza, por el principio biocéntrico y su marco teórico metodológico. Además, de sus estudios formales, ha recibido cursos y viajado para formarse de manera directa con maestras como Diana Milosevick (Dah Teatar, Serbia), Rosi Di Costanzo (Comuna Baires, Italia), Débora Hun (Puerto Rico) María Elena Garavelli (Argentina) Jutta Happekausen (Alemania) Pepa Díaz Meco (España) y profesores como Peter Shub (Alemania) Jango Edwards (EEUU) Laura Hertz (EEUU) Javier Pastor (España).
Davis crea La Barca Otro Teatro ${ }^{2}$ en el año 2009. La actriz y directora es experta en teatro espontáneo y trabaja con la técnica de improvisación y clown. Su escuela es un espacio de aprendizaje e innovación en el que, en el que recientemente ha incorporado estudios de biodanza. La directora decidió trabajar en el teatro puesto que encontró en ello una vía para expresarse: "Me apasiona el contacto con las personas y me defino, ante todo, como grupalista. Creo que ahí está mi especialidad, en la facilitación de grupos" (Davis, 2016).

En materia de teatro espontáneo los dos grupos que se han creado en Sevilla surgieron tras su aprendizaje con Davis. Cristina Domínguez Vázquez, quien se inició en esta disciplina con la actriz continuó su formación en Latinoamérica con su propia escuela (Transcrea) y línea didáctica (Teatro de Transformación). En Andalucía, las compañías más propulsoras se encuentran en Granada (La Tetera) y Sevilla (Improductivos y La Barca Otro Teatro). Además, la directora oferta técnicas innovadoras tales como el Impropainting, (variante del Soundpainting), creado por Walter Thompson, que consiste en la improvisación colectiva de artistas de diferentes disciplinas a partir de una dirección de tipo orquestal que se realiza en escena.

La empresaria es también una actriz experta en clown. Actualmente, investigadores se han detenido en analizar cómo repercute el clown y el teatro de improvisación en la enseñanza, entre ellos, en primer lugar podemos mencionar a Sánchez Velasco “En nuestra propuesta cooperativa, los estudiantes improvisan con su cuerpo ante una audiencia. Esta sirve al improvisador como espejo, pues en sus reacciones se proyecta un reflejo emocional de lo que el improvisador hace y dice" (2016: 39). Recientemente, Jara (2014) aborda la importancia del clown puesto que rescata la "autenticidad" del individuo:

Nos encontramos ante una forma de expresión y/o comunicación directa espontánea y primaria, con la que podremos recuperar el placer del juego, el dejarse llevar y los estados de máxima sensibilidad, en los que sentimos y reaccionamos más allá de los convencionalismos y las costumbres. Por todo esto y finalmente, podemos afirmar que el encuentro con nuestro clown supone una especie de sano viaje a lo más auténtico de cada persona (Jara, 2014: 18-19)

Muestra de ello, lo observamos también en el reciente estudio de Sánchez Velasco, en el que ha demostrado la importancia de la técnica de clown e improvisación en el ámbito de la competencia comunicativa:

2 La actriz decide llamar a su escuela La Barca, porque su propuesta consiste un viaje de un punto a otro, un espacio y un medio de crecimiento, no busca la retención del alumnado si no su emancipación y vuelo. Además, el nombre hace referencia a una bellísima frase que la acompaña hace muchos años "Naveguemos, el mar es invención de nuestra barca" (IBN AL BARUDI). 
El clown es la parte de nuestro ser más intuitiva puesto que no analiza, juzga, ni valora, solamente siente, se muestra y actúa espontáneamente. $Y$ además está en contacto con nuestra parte más espiritual, ya que vive profundamente su intimidad y se integra intuitivamente en su propio mundo emocional, para asi sentir, a su vez, el mundo que le (Sánchez Velasco, 2016: 20-21).

Al igual que ellos, la directora apuesta por esta disciplina que aumenta la creatividad, autoestima y habilidades sociales.

\section{LA INNOVACIÓN: ROTUNDA HISTORIA Y ESPONTAGAS}

La actriz ha impulsado la disciplina de la improvisación mediante la organización de festivales y participación en encuentros. Además, como muestra de ello, ha donado libros de improvisación y teatro espontáneo al centro de documentación de artes Galván. En esta obra, la artista apostó por la dignificación de la improvisación como arte teatral, que trasciende más allá del mero entretenimiento. Es destacable mencionar escénicas de Andalucía, puesto que no contaban con el suficiente material de referencia. Davis utiliza esta técnica porque la considera dinámica, cooperativa y creativa. El teatro de improvisación nos enseña a confiar en nuestras propias ideas y en las
de los demás, a lanzarse a lo desconocido, imagina, escuchar, crecer en las ideas
de otras personas, a trabajar todos a una para un objetivo común, a explorar
nuevos límites, a respetar y valorar a las otras personas (Davis, 2016).

La psicóloga social también apuesta por el clown. En su trayectoria ha observado que sólo en algunas convocatorias de festivales, ferias, diputaciones se incluye la categoría clown; sin embargo en ninguna se registra el teatro espontáneo, ni de improvisación. El clown, de historia masculina, ha comenzado una nueva etapa en España gracias a mujeres como Pepa Plana, Amaia Prieto, Pepa Díaz- Meco o Virginia Imaz fundadora de la compañía Oihulari Klown. Desde sus inicios el clown se transmitía de generación en generación, de padres a hijos. Desde que hace 30 años el clown abandonó la exclusividad de la pista de arena como espacio de presentación y se subió a los escenarios de los teatros, las mujeres comenzaron a formar parte del llamado movimiento del "Nuevo Clown". Tal y como afirma la actriz: "Las payasas aportan grandes dosis de ternura, se atreven a mirarse y a reflejar sus problemas y opiniones en escena. Las mujeres hemos avanzado en nuestra lucha por la igualdad pero aún no estamos en paridad" (Davis, 2016).

En sus espectáculos de teatro espontáneo reciben propuestas del público y las interpretan con música en directo. Entre las impulsoras del teatro espontáneo en España, primeramente podemos mencionar a Ana María Fernández en Salamanca (Entrespejos) y Davis en Sevilla (La Barca). A estas, se suman Alejandra Barbarellli en Madrid (Impronta) y Cristina Domínguez también en Sevilla (Transcrea). Se trata de un teatro de corte social donde priorizan el compromiso con las historias de la gente. La compañía Espontangas está compuesta íntegramente por mujeres y surge a partir de un grupo de alumnas de La Barca Otro Teatro, en el que Davis también se integró, como actriz y directora: “Cada una de las funciones se concibe como un juego de espejos, en el que los espectadores se reflejan unos en otros. Además, escuchamos las voces, anécdotas, sentimientos, recuerdos de los participantes en definitiva, rescatamos el valor de sus historias" (Davis, 2016). A la actriz le surge la idea de crear Rotunda Historia después de presenciar un maravilloso solo de improvisación de Omar Argentino que en España no es frecuente encontrar muchos solos de improvisación y aún menos interpretados por mujeres. Este proyecto surge porque la actriz anhelaba subirse al escenario inspirada en la magia del público, vivir el vértigo del salto al vacío y a la vez, acercarse a géneros literarios y estilos que trascendiesen la comedia. La intérprete confiesa que la implicación y la profesionalidad de Javier Durán (miembro de la compañía Improductivos) le hicieron que contara con su dirección. Durante la gestación se sumó Gregorio M. Toral, un maravilloso pianista que aceptó el reto de transformarse en músico improvisador.

Indiscutiblemente, llegar a tener éxito y poder vivir del teatro también tiene sus desventajas puesto que difícilmente podrá compaginar su vida profesional con la personal. La actriz reconoce que cuando se planteó la posibilidad de la maternidad, la idea le pareció totalmente incompatible con su trabajo: Ser emprendedora, en general y en artes escénicas en particular, me requería casi el $100 \%$
de mi tiempo. No sólo soy mujer, soy extranjera (sin contactos por familiaridad o historia) y
me dedico a disciplinas en desarrollo. Tengo a mi favor ser latinoamericana, es decir, estar
acostumbrada a contextos adversos, a la autogestión y falta de apoyo. Traigo entrenamiento
previo. En segundo lugar, los impuestos asfixian, no hay una política de ayudas ni fortalecimiento de la actividad cultural (Davis, 2016).

Esta falta de apoyo hace que "En líneas generales, [...] las mujeres artistas se sitúan en una encrucijada en la que se debaten entre priorizar su vida laboral o su vida familiar y personal mientras que los hombres no se enfrentan a estas situaciones" (Veiga Barrio, 2010: 67-68). El teatro es una profesión bastante sacrificada para las mujeres y difícilmente compatible con la vida familiar. La dramaturga y directora de escena Juana Escabias también reflexiona sobre la dificultad que supone vivir del teatro:

En la España del siglo XXI es complicadisimo. La crisis nos castiga. Por otro lado, quienes hacemos cultura estamos recibiendo un escarmiento político por parte del actual gobierno: que considera la cultura y el libre pensamiento enemigos personales" (Escabias en Martín Clavijo, 2014: 309). 
Como mujer y emprendedora, la actriz se ha enfrentado a numerosas dificultades para poder sacar adelante su proyecto empresarial teatral. Veiga Barrio explica que esta situación las conduce a experimentar una sensación de culpa, debido al modelo patriarcal impuesto por la sociedad:

Este sentimiento de culpabilidad, desde nuestro punto de vista, tendría una estrecha relación con el papel social que la sociedad asigna a las mujeres y lo que se espera de ellas. Ellas se

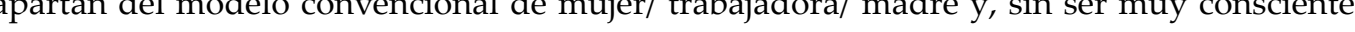
de este hecho, sus aspiraciones personales y profesionales chocan con las expectativas y tamientos que la sociedad patriarcal vuelca sobre las mujeres (Veiga Barrio, 2010: 105)

\section{EL TEATRO COMO ESTRATEGIA DE CONCIENCIACIÓN DE GÉNERO}

Las dramaturgas son las figuras que han logrado alcanzar más prestigio históricamente, según O'Connor "La notable corriente de dramaturgas que surge en el periodo democrático (desde 1978 hasta nuestros días) continúa hoy su curso ascendente [...]" (1978: 13). En España, en la dictadura franquista ${ }^{3}$ habían sufrido una discriminación tanto moral como política:

\section{La censura para las mujeres dramaturgas durante la dictadura franquista era doble, porque a la censura política se sumaba la moral. La sociedad española del momento no estaba preparada para asumir que la mujer dramaturga, tuviera derecho de dirigir un equipo de actores o la propia escena teatral (Vidal Egea, 2010: 64).}

Sin embargo, tras la dictadura franquista, afortunadamente la mujer gozó de mayor libertad: “El cambio de régimen político operado a la muerte del general Franco en 1975 y la emancipación de una sociedad tanto tiempo amordazada favorecieron la presencia

3 Es destacable mencionar que las adaptaciones teatrales de escritoras británicas también fueron censuradas, como el caso de Emily Brontë y su obra Cumbres Borrascosas. Aurelio Tejedor solicitó la petición de la obra teatral en 1943 que en un principio fue denegada: “En 1943 se registran cuatro ingresos: uno la solicitud [Exp.: 7911-43] de autorización de una adaptación teatral llevada a cabo po Aurelio Tejedor en colaboración con Arturo Guasch, solicitud que se deniega en primera instancia" (Pajares Infante, 2007: 59). Esta pequeña obra teatral finalmente vio la luz en 1944 en La Escena3 (pajalicación mensual de obras teatrales, en concreto es la número 71, publicada el 1 de diciembre de 1944 en Barcelona). La obra está dividida en tres actos y un epílogo; concretamente fue la última obra teatral publicada de la colección, el primer número se publica el 1 de abril de 1941 (Azcune, 2009: 21). Las publicaciones aparecían con una regularidad bastante rigurosa hasta el número 66 de la colección (15 de enero de 1944); a partir de este número las publicaciones fueron más irregulares. La Guerra Civil española (1936-39) interrumpió la publicación de las colecciones teatrales que aún aparecían en 1936. En los primeros años de la posguerra continúa la publicación de las comedias y es destacable mencionar que de las colecciones anteriores a la guerra solo sobrevivió Teatro Selecto -aunque el autor especifica que con algunos cambios- y desaparecieron Teatro Frívolo y La Farsa. Sin embargo aparecieron algunas novedosas colecciones como Talía, La Escena, Biblioteca Teatral, La farándula, Las Máscaras, Proscenio, Éxito y Teatro (Azcune, 2009: 14). Según este autor la nueva publicación de estas obras teatrales era una señal evidente de la importancia que tenía el teatro aún en aquella época. de las individualidades y con ello se concedió voz pública a la mujer" (Virtudes Serrano, 2004: 562). Es destacable mencionar que un gran número de dramaturgas han intentado concienciar a la sociedad sobre la importancia de la violencia de género. Entre ellas, en primer lugar debemos hacer referencia a la poetisa Patricia Ariza, que según afirma Bolaños es: “[...] una de las directoras y dramaturgas que ha impulsado el teatro de género en Colombia, y en especial ha sido una colaboradora incondiciona con el Teatro La Máscara" (2010: 39). En segundo lugar, también se debe mencionar a grandes dramaturgas como Paloma Pedro que en su obra, La noche que ilumina (1995) denuncia el abuso diario al que muchas mujeres están sometidas:

[...] las mujeres en situación de violencia de género son atacadas a diario, no a causa de las drogas, el alcohol o una supuesta patología de los agresores, sino porque sus compañeros o excompañeros han heredado un rol de dominación androcéntrico y discriminatorio de la mujer (García-Pascual, 2010: 264).

Otro ejemplo es el estudio de Dominique Moreno con su obra Roles femeninos en el teatro contemporáneo español. Construcciones desde la violencia de género (de 2002 a 2012 (Pascual, sin fecha) que fue galardonada con el Premio Leonor de Guzmán de la Universidad de Córdoba. La prestigiosa dramaturga Itziar Pascual también apuesta por la defensa de la igualdad de género en Pared, cuya producción obtuvo el IV premio de Teatro Madrid Sur (2004) (Cordone, sin fecha). Pared (2004), de Pascual, clama por la consideración de esta violencia como delito público, a una de sus coprotagonistas, no sólo maltratada por su marido, sino también por su hijo García- Pascual, 2010: 264)

En esta búsqueda por la denuncia al maltrato, también han surgido adaptaciones teatrales, tales como La historia de amor del siglo de la escritora filandesa Märta Tikkanen que se publicó por primera vez en 1978. Numerosas representaciones se han basado en el poemario que cuenta la historia de una mujer y a la vez de muchas, en su recorrido como parejas de un amor alcohólico y violento.

Davis también ha participado en esta transformación social con los siguiente espectáculos. En primer lugar representó con la compañía sevillana Dos Luna Teatro La travesía de la dama blanca, dirigida por Mariana González (2008) en la que afronta la violencia de género mediante un lenguaje gestual.

Tengo este recuerdo grabado: recibía a mi compañera, la cargaba sobre mi espalda para levarla a casa y la cuidaba hasta que se recuperaba. Más tarde, la vela regar las poquitas . 
La representación de esta performance siempre la conducía a un estado de confusión (a veces se quedaba sentada pensativa, llorando o temblando después de la actuación). Su personaje era la acompañante que permanecía cerca de una mujer que, desgraciadamente y como ocurre en la vida real, entra en el círculo de la violencia y padece una situación de aislamiento de la que no es capaz de salir: También junto a esta compañía representó Las tres flores de la costa, dirigida por David Fernández (2009), en la que a través de un lenguaje humorístico abordaron la masculinización de la mujer como camino de poder y reconocimiento y también dificultades de distinta índole que debe afrontar.

En segundo lugar, nos detendremos en su performance con Páginas Violeta. La actriz conoció a Teresa Alba, la presidenta de Páginas Violeta en una función de Rotunda Historia. Esta asociación ha sido el apoyo más importante que ha recibido hasta el momento con quienes ha producido el espectáculo Linaje. Otra mirada sobre la violencia de género. A Teresa Alba le propusieron montar un espectáculo contra la violencia de género a partir de su texto "Elegía por las mujeres muertas" y contó con su participación Linaje nace de la desesperación de las mujeres y actriz define la función como "un grito desgarrado, impotente, desolador, que me habita en las entrañas, en una maraña de recuerdos y de marcas invisibles" (Davis, 2016). Con el propósito de atenuar el horror de la violencia sintió la necesidad de dotar de belleza a la obra e insertó danza. El espectáculo contó con la colaboración Mercedes Velazco Rubio y ambas construyeron un camino entre la realidad y la ficción que narra la historia de Antonia desde su bisabuela, su abuela y su madre, hasta llegar a ella. El espectador se enfrenta a la mirada de la niña que observa, que vive una experiencia traumática, oye golpes, ve a su madre lastimada, vive con miedo. El personaje de Mercedes simboliza la fuerza interior de Antonia y a la vez la de todas las mujeres; en definitiva, el espectáculo refleja el modo en el que los adultos abordan la violencia de género.

\section{La obra es un testimonio y sobre todo, un acto de liberación, un grito que busca estremecer los cimientos del horror. En ocasiones desconocemos su origen e incluso podemos tener formas de violencia internalizadas (hacia nosotras mismas u otras personas) y no darnos cuenta (Davis, 2016).}

\subsection{El género desde el humor: A Todo Trapo}

4 Cada vez hay un mayor número de asociaciones como Páginas Violeta que lucha por la igualdad de oportunidades y ha puesto en marcha la plataforma Escenaria con el propósito de difundir el trabajo de las mujeres.

5 Se puede visionar Linaje en https://www.youtube.com/watch?v=jHJqDzgLt4E

6 El texto había sido recitado por la prestigiosa actriz Carmen Troncoso y se había puesto en escena con anterioridad en Flores de hierro.
Davis es la autora de $A$ todo trapo, una obra que refleja la vida polifacética de la gran parte de las mujeres de clase media. En todo momento, la actriz alterna el clown y el humor y su propósito es "mostrar las contradicciones de la mujer en la edad adulta y los diferentes estados de ánimo" (Davis, 2016). Con el paso de los años, algunos personajes fueron cambiando y al llegar a España, dos de ellos desaparecieron del espectáculo, puesto que no encajaban en el imaginario colectivo local. El lenguaje también sufrió modificaciones teniendo en cuenta que las variedades lingüísticas del argentino y andaluz:

\section{A todo trapo es la obra teatral que más satisfacciones me ha dado. Me acompaña hace 16}

lenguaje, de personajes, de etapas de mi vida, de espacios, de equipo técnico (Davis, 2016).

En este espectáculo, Davis reflexiona sobre la capacidad del teatro como espacio para la deconstrucción y la reconstrucción de los géneros, como vehículo para el encuentro entre personas más allá de la identidad sexual de cada una y como herramienta para la comunicación y la transformación en un momento de tanta efervescencia social como el actual.

Interpreto a una mujer trabajadora que, al mismo tiempo, sueña con el amor Creo que hasta la más fuerte e independiente de las mujeres sigue esperando al amor de su vida. Nosotras, machista [. ] tiene que llegar el príncipe azul, tenemos que ser buenas amas de casa (Davis, 2016).

Ella cree que podemos enfrentarnos a esta realidad impuesta por la sociedad patriarcal con humor. En $A$ todo trapo, se ríe de mí misma, permite que el espectador también lo haga y así todos se van encontrando en una u otra de las facetas que presenta el espectáculo:

Mi espectáculo $A$ todo trapo me permite más que hablar de género en el teatro hablar de género desde el humor. Siempre me defino más como "grupalista" "humorista". No puedo decir que lo que yo hago es típico de las mujista" o "humorista". No puedo decir que lo que yo hago es tipico de las mujeres, porque las mujeres hacen todo tipo de teatro, resultado de una larga lucha. Antes las mujeres siempre ocupábamos papeles muy estigmatizados y ahora, desde que somos dramaturgas y directoras y no sólo actrices, por un lado han cambiado los criterios en el casting -y ya no hay que seducir necesariamente al director, podemos juzgar directamente la capacidad de la intérprete- $y$, por otro, podemos contar cosas, cosas que no solamente nos interesan a nosotras, sino que interesan a todas y todos, pero desde una mirada femenina (Davis, 2016).

El clown está también presente en la obra. Davis encuentra un paralelismo entre el lugar histórico de la mujer en la corte y el del bufón. La mujer, como el bufón, es quien más ha sufrido en esta sociedad en la que siempre se la relegó a una posición marginal censuraron su palabra y anularon su ser. 
¿Cuántas mujeres -y me gustaría hablar de ello en pasado, pero sigue siendo presente- al levantar la voz son ridiculizadas, anuladas? ¿Cuantas fueron quemadas en el pasado? Lo médicos eran médicos y las médicos eran brujas. Curaban con lo mismo, hacían lo mismo, pero las brujas acababan en la hoguera. Era una forma de silenciarnos. Por eso el riesgo de la mujer ha sido el mismo que el del bufón: salir de ese pantano, de ese destierro, de esa nada que es peor que la nada, de ese castigo, y volver a pasar por el castigo. Las mujeres pasaron por ello dura

La mujer por haber sobrevivido a ello, por haber ido hacia delante, posee una gran fortaleza. Falcón O’Neil define las restricciones que siguen padeciendo las mujeres en la sociedad actual: Esta es la gran conquista que logran las mujeres después de doscientos años de luchas: la
libertad de independizarse de la tutela y opresión del padre, del marido, del hijo. Una vez
rotas las cadenas, la libertad les supone encontrarse a campo abierto, sin amo, pero también
sin protección, en un mundo masculino dominado por la desigualdad en el reparto de la
riqueza, por la competencia laboral y la agresividad machista, pero manteniendo sus obligaciones reproductoras y de trabajadoras domésticas (Falcón O'Neil, 2014: 55).

La realidad que describe Falcón O'Neil se expone en A Todo Trapo. Davis interpreta a la mujer actual, que tiene un trabajo de ocho horas; si no tiene hijos, se ve obligada a trabajar diez o doce, y si los tiene, cuando sale del trabajo debe, además, ser madre y compartirlo con el trabajo del hogar. Como actriz y autora reconoce que la obra desprende humor femenino y que no concibe el hecho de crear una obra sin que su mirada de mujer permanezca impresa: “No creo que ninguna una actriz pueda representar una obra que no la refleje, sería una falacia. Aunque interpretes Hamlet, estás ahí, porque el Hamlet que tú interpreta te refleja y porque nunca hay dos Hamlets iguales o dos Antígonas o Bernardas" (Davis, 2016). Como mujer, ha demostrado poseer sensibilidad y fortaleza suficiente para mostrar su propia mirada ante la igualdad de género, aunque desgraciadamente haya crecido y trabajado en ambientes machistas durante mucho tiempo.

\section{No creo que los hombres no sean sensibles, creo que no están educados para reconocer esa sensibilidad, porque lo social no se lo permite y porque los medios de comunicación no hacen más que reforzar permanentemente esa etiqueta de masculinidad, de la misma manera que imponen una mirada fenenina que es trágilo superficial. No obstante, si las mujeres pudimos dar el salto, ahora les toca a los hombres darlo. Somos las generaciones que más estamo viviendo la contradicción y la lucha dialé construir nuestra feminidad (Davis, 2016)}

Sin embargo, existe la posibilidad de afrontar esta realidad con humor. En $A$ todo trapo, cuando se ríe de sí misma, permite que el espectador también se ría y así se encuentran en una $u$ otra de las facetas que presenta el espectáculo. "Las mujeres siempre hemos mostrado una gran capacidad de complicidad silenciosa, porque históricamente nuestra complicidad ha sido silenciada" (Davis, 2016).
Desgraciadamente, numerosos casos de mujeres se "invisibilizaron" como medida para sobrevivir lo cual no quiere decir que se colocaran en una postura real de sumisión.

A lo largo de la historia, las mujeres, en ese impulso por sobrevivir, han generado códigos de encuentro. Me viene ahora a la mente la imagen de las mujeres bordando juntas, preparando entre ellas y no podían ni imaginar lo que ocurría más allá del silencio [...] (Davis, 2016)

Actualmente, contamos con medios de comunicación más abiertos, tenemos más posibilidad de ser visibles y además tenemos a nuestra disposición la herramienta del humor.

El humor es una forma de resistencia y de reflexión. A menudo me veo en la necesidad de aclarar que mi forma de ver el teatro, el teatro que yo hago, no quiere decir que no crea

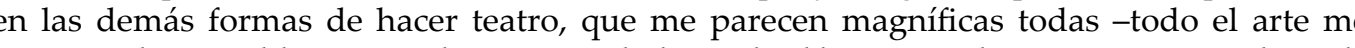
parece indispensable para cualquier sociedad. Desde el humor podemos generar un clima de confianza con el espectador (Davis, 2016)

Además del humor, Davis trabaja con un formato reducido puesto que le permite un acercamiento con el público y ese contacto es lo que en realidad nutre al espectáculo:

\section{En el espectáculo se genera un espacio íntimo donde me río a la vez que muestro mis desgracias -y me desnudo ante el espectador, para ello es necesario generar un clima de que genero un juego de confianza con el espectador en el que el que este disfruta (Davis,} 2016).

La actriz siente que al revelar al público su condición sexual se crea automáticamente una barrera con parte de él. Entonces, intenta desnudarse aún más: les narra su vida y cómo la ha vivido e incluso les hace partícipes de su primera experiencia con una mujer. Experiencia que ella la califica de extraña y es el público quien le añade connotaciones despectivas. Se puede considerar esta cuestión desde la igualdad pero también es importante recordar que todo ello nace de un ejercicio de confianza.

El mundo real no existe pero existe nuestra mirada sobre el. De modo que yo trabajo en mi mundo real a la vez que creo un juego de confianza con el espectador en el que este disfruta. Ojalá llegué el día que un hombre o una mujer heterosexual, bisexual o pentasexual (Davis, 2016)

\section{CONCLUSIONES}

Indiscutiblemente, con el paso del tiempo el trabajo de las dramaturgas ha sido más valorado por la sociedad; no obstante, otras profesionales no han logrado alcanzar su merecido reconocimiento. En primer lugar, nos hemos detenido en analizar el caso de una psicóloga social de origen argentino que gracias a su esfuerzo ha logrado poner 
en marcha su propia compañia de teatral, la Barca Otro Teatro de la que surge una segunda: Espontagas. La directora trabaja con la técnica de improvisación y clown que mejora las competencias comunicativas y sociales del alumnado. En segundo lugar hemos analizado su faceta artística con la que pretende concienciar a la sociedad sobre la importancia de la educación en la igualdad de género. En este caso, nos hemos detenido en su trabajo en la compañía Dos Lunas Teatro con la producción de La travesía de la dama blanca (2008) y Las tres flores de la costa (2009). Como autora de la obra $A$ Todo Trapo Davis muestra su propia mirada ante la igualdad de género en una época de transformación social. En definitiva, hemos pretendido visibilizar la trayectoria profesional de Davis para que su trabajo, como el de otras muchas mujeres en las artes escénicas sea valorado

\section{REFERENCIAS BIBLIOGRÁFICAS}

Azcune, V., Las pequeñas colecciones teatrales de posguerra. Madrid: Consejo Superior de Investigaciones Científicas (CSIC), 2009.

Bolaños, L., "Patricia Ariza: una polifonía de resistencia artística”, Lenguajes teatrales Encuentro de Mujeres de Iberoamérica en las Artes Escénicas. Cádiz, Dora Sales, 2010, pp. 35-39.

Cenizo Rodríguez, M., Moral del Arroyo, G., Varo Baena, R., “El teatro como medio de sensibilización contra la violencia de género en la adolescencia (Estudio exploratorio sobre el uso de la obra de teatro Ante el espejo como herramienta de prevención y sensibilización", Stichomythia: Revista de teatro español contemporáneo, 11-12, 2011, pp. 255-267.

Cordone, G., Cuerpos reales y cuerpos virtuales en Pared, de Itziar Pascual. Internet 01-06-2016. <http://parnaseo2.uv.es/Ars/Autores/Pascual/obras/Cordone.pdf> Davis, P., Entrevista concedida inédita, 2016.

Dora Sales, D., El teatro, campo de fuerza y juego experimental, Encuentro de Mujeres de Iberoamérica en las Artes Escénicas. Cádiz: Festival Iberoamericano de Teatro de Cádiz, 2014.

---- El compromiso a escena. Cádiz, Festival Iberoamericano de Teatro de Cádiz Encuentro de Mujeres de Iberoamérica en las Artes Escénicas (12-2008, Cádiz) Madrid: Ediciones y Publicaciones Autor, 2009.

Falcón, L., Los nuevos machismos. Barcelona, Editorial UOC, 2014

Fernández Morales, M. El teatro como práctica ideológica. Violencia de génerol violencia política en la escena estadounidense contemporánea. España: Ediuno Universidad de Oviedo, 2005
García-Pascual, R., “Sensibilización y denuncia de malos tratos en el teatro español contemporáneo: Paloma Pedrero e Itziar Pascual", Revista Anagnórisis, 1, 2010.

Jara, J., El clown, un navegante de las emociones. Barcelona: Octaedro, 2014

Laferrière, G., La pedagogía puesta en escena. Ciudad Real: Ñaque, 1997.

Martín Clavijo, M., “Entrevista a Juana Escabias., dramaturga en escena”, Raudem, Revista de Estudios de las Mujeres, II (2014), pp. 305-322. Internet. 15 -06-2016<http:// www.anagnorisis.es/pdfs/Raquel_Garcia-Pascual.pdf.>

O'Connor, P. W., One-act Spanish plays by women about women. Mujeres Sobre Mujeres, Teatro Breve Español. Edición bilingüe. Traducción de Patricia Walker O'Connor Madrid: Fundamentos, 1978

Páginas Violeta (Asociación). Internet <http://paginasvioleta.org/>.

Pajares Infante, E., “Traducción y censura: Cumbres borrascosas en la dictadura franquista", Traducción y censura en España (1939-1985). Estudios sobre corpus TRACE: cine, narrativa, teatro. Bilbao: Universidad del País Vasco; Universidad de León, 2007. Internet 11-06-2016. <https://addi.ehu.es/bitstream/10810/10159/4/PAJARES\%20 INFANTE,\%20E_Trad ucci\%C3\%B3n\%20y\%20censura.pdf.>, pp 49-103.

Proaño, L., Espacios de representación, Encuentro de Mujeres de Iberoamérica en las Artes Escénicas, Cádiz, Festival Iberoamericano de Teatro de Cádiz 2006.

Sánchez Velasco, A., La Dramatización como Estrategia para la Mejora de la Competencia Comunicativa: Una Investigación en Educación Secundaria desde las Técnicas de Improvisación y Clown. Murcia, Universidad de Murcia (2016). Internet. 22-052016. <http://www.tdx.cat/bitstream/handle/10803/366514/TASV.pdf?sequence=1>

Serrano, V. “Dramaturgia femenina fin de siglo. Estado de la cuestión”, Arbor CLXXVII, 699-700 (Marzo-Abril 2004), 561-572 pp. Internet. 18-06-2016. <http://arbor.revistas. csic.es/index.php/arbor/article/view/595/597>. 